\title{
Intraperitoneal Glucose Sensing is Sometimes Surprisingly Rapid
}
A.L. Fougner 12
K. Kölle ${ }^{12}$
N.K. Skjærvold ${ }^{3}$ N.-A. L. Elvemo 5
D.R. Hjelme ${ }^{6}$
R. Ellingsen ${ }^{6}$ S.M. Carlsen ${ }^{4} 7$
Ø. Stavdahl ${ }^{1}$

\author{
${ }^{1}$ Dept. Engineering Cybernetics, Norwegian University of Science and Technology (NTNU), Trondheim, Norway \\ ${ }^{2}$ Central Norway Regional Health Authority, Trondheim, Norway \\ ${ }^{3}$ Dept. Circulation and Medical Imaging, NTNU, Trondheim, Norway \\ ${ }^{4}$ St Olavs Hospital, Trondheim, Norway \\ ${ }^{5}$ GlucoSet AS (NO 997780 922) \\ ${ }^{6}$ Dept. Electronics and Telecommunication, NTNU, Trondheim, Norway \\ ${ }^{7}$ Dept. Cancer Research and Molecular Medicine, NTNU, Trondheim, Norway
}

\begin{abstract}
Rapid, accurate and robust glucose measurements are needed to make a safe artificial pancreas for the treatment of diabetes mellitus type 1 and 2. The present gold standard of continuous glucose sensing, subcutaneous (SC) glucose sensing, has been claimed to have slow response and poor robustness towards local tissue changes such as mechanical pressure, temperature changes, etc. The present study aimed at quantifying glucose dynamics from central circulation to intraperitoneal (IP) sensor sites, as an alternative to the SC location.

Intraarterial (IA) and IP sensors were tested in three anaesthetized non-diabetic pigs during experiments with intravenous infusion of glucose boluses, enforcing rapid glucose level excursions in the range 70-360 mg/dL (approximately 3.8-20 mmol/L). Optical interferometric sensors were used for IA and IP measurements. A first-order dynamic model with time delay was fitted to the data after compensating for sensor dynamics. Additionally, off-the-shelf Medtronic Enlite sensors were used for illustration of SC glucose sensing.

The time delay in glucose excursions from central circulation (IA) to IP sensor location was found to be in the range 0-26 s (median: $8.5 \mathrm{~s}$, mean: $9.7 \mathrm{~s}$, SD $9.5 \mathrm{~s}$ ), and the time constant was found to be 0.5-10.2 $\mathrm{min}$ (median: $4.8 \mathrm{~min}$, mean: $4.7 \mathrm{~min}$, SD $2.9 \mathrm{~min}$ ).

IP glucose sensing sites have a substantially faster and more distinctive response than SC sites when sensor dynamics is ignored, and the peritoneal fluid reacts even faster to changes in intravascular glucose levels than reported in previous animal studies.

This study may provide a benchmark for future, rapid IP glucose sensors.
\end{abstract}

Keywords: Diabetes, Artificial Pancreas, Closed-loop systems, Type 1 diabetes, Type 2 diabetes.

\section{Introduction}

Glucose concentration can in principle be measured in all tissues. While intraarterial (IA) and intravenous (IV) measurements are not practically usable in outpatients, subcutaneous (SC) sensing has become the standard for continuous glucose monitoring (CGM) during the last 15 years. However, state of the art SC glucose sensing technologies with enzyme-based amperometric technology still face challenges with time delays and slow dynamics (Basu et al., 2013; Cengiz and Tamborlane, 2009; Boyne et al., 2003; Davey et al., 2010) as well as poor robustness (Helton et al., 2011; Mensh et al., 2013), even though recent publications 
indicate that novel CGM systems may be less prone to such problems (Garcia et al., 2013; Bailey et al., 2015). These challenges originate partly from the sensor technology and partly from the physiologically slow glucose transfer between intravascular glucose levels and glucose levels in subcutaneous tissue.

Intraperitoneal (IP) glucose sensing seems to offer faster dynamics (including shorter time delays) than $\mathrm{SC}$ sites (the term dynamics/system dynamics is hereafter used synonymously with what has previously been denoted kinetics, e.g. by Burnett et al. (2014)). Additionally, an IP sensor location may be less prone to mechanical disturbances (Helton et al., 2011; Mensh et al., 2013), variation due to fluctuation in tissue perfusion (Cengiz and Tamborlane, 2009; Burnett et al., 2014; Stout et al., 2004) and temperature effects as the body core temperature is strictly regulated. Thus, based on previous animal studies, the peritoneal cavity is a promising alternative location for continuous glucose sensing (Burnett et al., 2014; Clark et al., 1987, 1988; Velho et al., 1989).

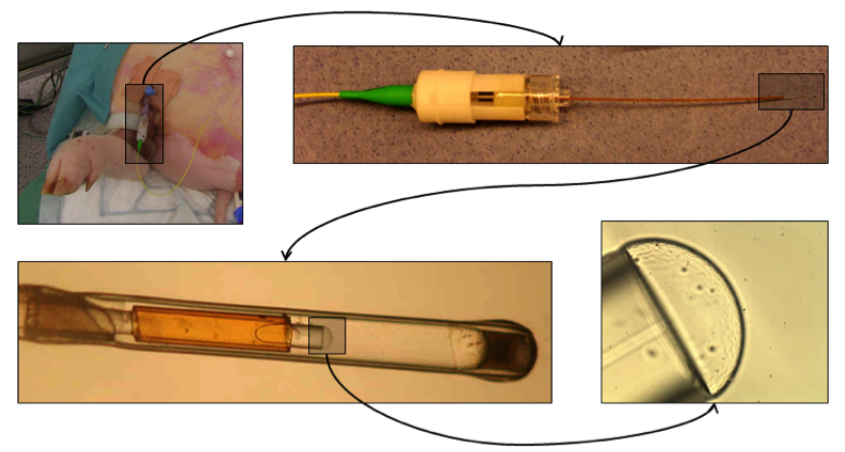

Figure 1: The GlucoSet sensor (Tierney et al., 2009; Skjærvold et al., 2012).

\subsection{Objectives}

The aim of the present study was to (A) assess whether interferometric sensors can be used to measure intraperitoneal (IP) glucose levels, and to (B) quantify how fast the glucose dynamics are from central circulation to IP sensor sites. We consider (B) to be the most important of these, but $(\mathrm{A})$ is a requirement in order to perform $(\mathrm{B})$.

\section{Methods}

The study was approved by the Norwegian Animal Research Authority (FOTS approval id 6538).

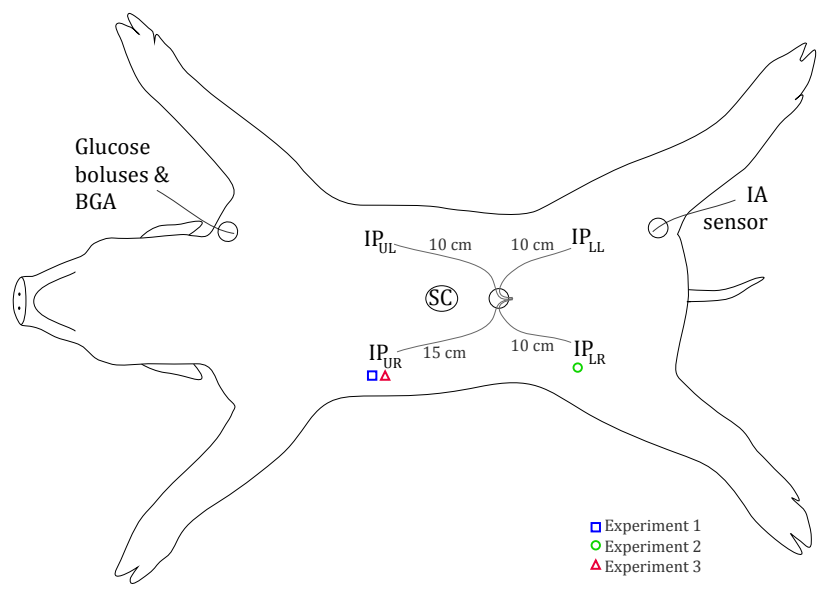

Figure 2: Approximate sensor placement and other instrumentation on the animal $(\mathrm{BGA}=$ blood gas analysis; IP = intraperitoneal; IA = intraarterial; $\mathrm{SC}=$ subcutaneous, $\mathrm{UL}=$ upper left quadrant, UR = upper right quad., $\mathrm{LL}=$ lower left quad., $\mathrm{LR}=$ lower right quad.). The working IP sensors for each experiment is indicated with symbols. The figure is licensed under a Creative Commons BY-NC-SA 4.0 license (hereafter abbreviated to Copyright: CC BY-NC-SA 4.0).

\subsection{The animal model}

Three healthy, young farm pigs, weight 25-30 kg, were enrolled in the study and used in one experiment each, hereafter named experiment 1,2 and 3. The animals were anaesthetized and euthanized as described in earlier studies (Skjærvold et al., 2012, 2013). A central venous line was established via the left internal jugular vein and an arterial line via the left external carotid artery, respectively, for monitoring, glucose bolus administration and blood sampling.

\subsection{Sensors}

For IA and IP glucose sensing, optical interferometric sensors from GlucoSet (GlucoSet AS, Trondheim, Norway) were used. The GlucoSet sensors are designed for intravascular use, and were at an experimental stage at the time of the current experiments. The sensor relies on interferometric measurements of the length change in a sphere-shaped hydrogel on the tip of an optical fiber (see Figure 1). Due to the hydrogel's composition it contracts or expands reversibly depending on the glucose concentrations. This length change is measured with the interferometric technique. The sensor version is named "3APB-alpha" and has previously been described (Tierney et al., 2009; Skjærvold et al., 2013). 
A modification of the original 3APB-alpha was used in one experiment, hereafter referred to as "3APB-beta". This modified sensor has slower dynamics but is more robust to other constituents of the measured fluid. Details on the specific composition are proprietary to the sensor manufacturer.

Enzyme-based amperometric Enlite sensors from Medtronic (Medtronic PLC, Dublin, Ireland) were used for SC glucose measurements, since the GlucoSet sensors cannot be used subcutaneously. These sensors were used only for illustration purposes, as their data was too sparse for system identification in the short segments analysed (offering values only every 5 minutes).

Blood samples from the external carotid artery were used for reference glucose measurements and analysed on a Radiometer ABL 725 blood gas analyser (Radiometer Medical ApS, Brønshøj, Denmark). During experiment 1 , these samples were taken mostly in stable periods (i.e. before each glucose bolus). During experiment 2 and 3 , blood samples were taken more frequently, especially after each glucose bolus, in order to verify the IA glucose level excursions.

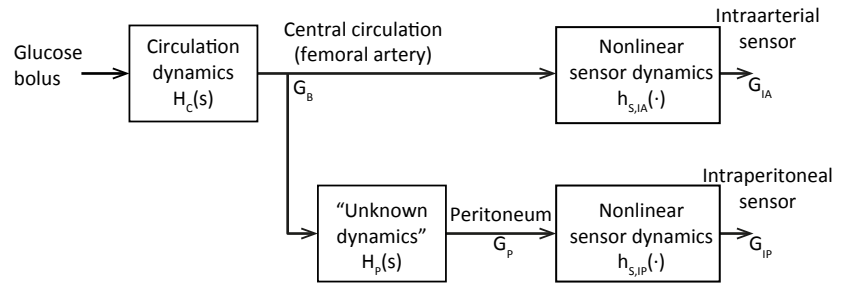

Figure 3: Model of glucose dynamics from infusion site to sensor sites, where $\mathrm{G}_{\mathrm{B}}(t)$ is the blood glucose concentration; $\mathrm{G}_{\mathrm{P}}(t)$ is the peritoneal fluid (or peritoneal lining) glucose concentration; $\mathrm{G}_{\mathrm{IA}}(t)$ is the intraarterial (IA) sensor signal; $\mathrm{G}_{\mathrm{IP}}(t)$ is the intraperitoneal (IP) sensor signal; $\mathrm{H}_{\mathrm{C}}(s)$ is the circulation dynamics (from jugular vein to the femoral artery); $\mathrm{H}_{\mathrm{P}}(s)$ is the unknown physiological dynamics (from the femoral artery to the peritoneal sensor site); and $\mathrm{h}_{\mathrm{S}, \mathrm{IA}}(\cdot), \mathrm{h}_{\mathrm{S}, \mathrm{IP}}(\cdot)$ are the nonlinear sensor dynamics in IA and IP sensors, respectively. Copyright: CC BY-NC-SA 4.0.

\subsection{Sensor placement}

The intention was to use one IA and one IP sensor in each experiment. However, a larger pool of sensors was used in order to have some in backup, since the interferometric sensors are susceptible to damage during insertion. Additionally, the IP sensor response can vary between different locations in peritoneum, so they were directed in four different angles from the insertion. Some sensors were corrupted by noise or other artifacts to the point where the glucose boluses were completely obscured and were thus not interpreted as a measure of glucose levels (hereafter called "noninterpretable sensors"). In the end only one IA and one IP sensor worked in each experiment, so a complete analysis of sensor location was not carried out. See also the discussion in Section 4.

Approximate sensor placement is shown in Figure 2. IP glucose sensors were inserted through a minilaparotomy in the ventral midline a few centimetres below the umbilicus, and the sensors were directed towards different IP positions. IA glucose sensors were inserted through the superficial part of the femoral artery after minimal surgical cut-down, either bilaterally (experiment 1 and 2) or uni-laterally (experiment 3 ). In experiment 1 , one $3 \mathrm{APB}$-alpha sensor and one 3APB-beta sensor were tested, but only the 3APBalpha signal was used for system identification (see Section 2.5.2). In experiment 2, the 3APB-alpha sensor was damaged during insertion, so only the 3APB-beta sensor was used. In experiment 3, one 3APB-alpha sensor was used.

During experiment 1, two IP sensors were inserted, but one of them was damaged during insertion, so only one IP sensor worked ( $15 \mathrm{~cm}$ into the upper right quadrant, see Figure 2). Accordingly, in experiment 2, the number of IP sensors was increased to four. All sensors gave readable signals, but only one of them $(10 \mathrm{~cm}$ into lower right quadrant) could be interpreted as a measure of glucose levels. The signals from the remaining sensors were deemed noninterpretable.

During experiment 3, three IP sensors were inserted. It was decided to move/pull on the sensors to see if a new position or a change to the sensor surroundings influenced the signal. One sensor $(15 \mathrm{~cm}$ into the upper right quadrant) worked during the whole experiment, but dynamics were changed after pulling it $1 \mathrm{~cm}$ between bolus segments C and D (see Section 3 and Table 1). The other two sensors, placed $15 \mathrm{~cm}$ into the lower and upper left quadrants, respectively, did not give usable signals even after moving them to new locations (one was moved to the lower right quadrant) or pulling them 1-5 centimetres. These were deemed noninterpretable.

All disturbances (e.g. unintentionally touching a sensor cable) were recorded, in order to be able to interpret the signals and noise.

In summary, one IP sensor worked in each experiment. Their placements were $15 \mathrm{~cm}$ into the upper right quadrant (experiment 1), $10 \mathrm{~cm}$ into the lower right quadrant (experiment 2) and $15 \mathrm{~cm}$ into the upper right quadrant (experiment 3), respectively. 
SC sensors were placed according to manufacturer's instructions in the ventral midline approximately five centimetres above the umbilicus.

\subsection{Glucose bolus administration}

Glucose boluses were administered as 2, 4 and $8 \mathrm{~mL}$ of glucose $500 \mathrm{mg} / \mathrm{mL}$ (B. Braun, Melsungen, Germany), yielding boluses of 1,2 and $4 \mathrm{~g}$ or $5.5,11.1$ and $22.2 \mathrm{mmol}$. The boluses were injected into the external jugular vein within $2-3$ seconds and the catheter was immediately flushed with $10 \mathrm{~mL}$ of $\mathrm{NaCl} 9 \mathrm{mg} / \mathrm{mL}$. Boluses were injected 10--20 minutes apart.

The first glucose bolus of each trial was initiated only after completion of SC sensor calibration and insertion of IP sensors.

As the last part of each experiment, glucose was injected in series of 3-4 boluses with approximately two minutes in between.

\subsection{Synchronization and data handling}

Every recorded action, such as glucose boluses or blood sampling, was time stamped. During start-up of each experiment, a synchronization procedure was performed. Since the SC sensor read-out unit (Medtronic Guardian Real-Time CGM) does not indicate seconds, timestamps from all other sensors were taken when the minute indicator incremented on the Medtronic device, yielding second-level synchronization across the different devices.

Before system identification, data was segmented so that each segment started with a glucose bolus and ended before the next known bolus or recorded disturbance. Hereafter, segments are named by combining the experiment number $(1-3)$ with capital letters, e.g. segment 3F representing "experiment 3, segment F". Segments with interruptions too soon after the glucose bolus, e.g. before the IA glucose level had returned to within $18 \mathrm{mg} / \mathrm{dL}(1 \mathrm{mmol} / \mathrm{L})$ above the baseline level, were not used in the subsequent analysis. Segments with no functioning IP glucose sensors were also excluded from the analysis. In total, 8 of 21 segments were excluded from analysis.

\subsubsection{Sensor calibration}

Prior to each experiment, the sensors were calibrated in a three-point procedure using calibration fluids with glucose concentrations of 0,72 and $216 \mathrm{mg} / \mathrm{dL}(0,4$ and $12 \mathrm{mmol} / \mathrm{L})$. Before a sensor was inserted into the animal, it was held into each fluid at $37^{\circ}$ until the signal had stabilized. Based on the known glucose concentrations and the resulting raw sensor signal, the three free parameters in the sensor signal functions (cf.
Eqs. 5 and 6 below) were estimated using least squares fit. The resulting sensor transfer function was subsequently inverted in order to compute glucose concentration from the raw sensor signals.

SC sensors were calibrated two hours after insertion; at a steady glucose level before any glucose bolus had been given, using IV blood analysed on the blood gas analyser for reference measurement.

\subsubsection{Modelling and system identification}

The block diagram of Figure 3 illustrates the mathematical model of glucose transport from the glucose bolus infusion at the jugular vein to the sensor sites in the peritoneal cavity and in the femoral artery. The dynamics indicated are $\mathrm{H}_{\mathrm{C}}(s)$, the circulation dynamics (from jugular vein to the femoral artery); $\mathrm{H}_{\mathrm{P}}(s)$, the unknown physiological dynamics (from the femoral artery to the peritoneal sensor site); and $\mathrm{h}_{\mathrm{S}, \mathrm{IA}}(\cdot)$, $\mathrm{h}_{\mathrm{S}, \mathrm{IP}}(\cdot)$, nonlinear sensor dynamics of IA and IP sensors, respectively. The symbol $s$ represents the Laplace variable, and the symbol "." is used for the latter two functions to signify the measured variable, i.e. $\mathrm{G}_{\mathrm{B}}(s)$, the blood glucose concentration, and $\mathrm{G}_{\mathrm{P}}(s)$, the peritoneal fluid (or peritoneal lining) glucose concentration.

A system identification procedure was conducted in order to quantify $\mathrm{H}_{\mathrm{P}}(s)$ and the time to reach half of the maximum measured IP sensor signal after a glucose bolus (denoted $t_{\max / 2}$ ). In order to find $t_{\max / 2}$, it was also necessary to estimate the circulation dynamics, $\mathrm{H}_{\mathrm{C}}(s)$.

The following equation defines the model of $\mathrm{H}_{\mathrm{P}}(s)$, consisting of a time delay and a linear transfer function, as previously done by e.g. Burnett et al. (2014):

$$
\mathrm{H}_{\mathrm{P}}(s)=\frac{\mathrm{K}}{1+\mathrm{T} s} e^{-\tau s}
$$

The IP sensors were calibrated manually with respect to gain and offset, and the sensor elements are manually fabricated (so that parameter $K$ is different for every set of IA and IP sensors). Thus, only the time constant $\mathrm{T}$ and the delay $\tau$ are reported as results.

From all experiments, $\mathrm{G}_{\mathrm{IA}}(s)$ (IA sensor signal) and $\mathrm{G}_{\mathrm{IP}}(s)$ (IP sensor signal) are used to estimate $\mathrm{H}_{\mathrm{P}}(s)$ using the following equations:

$$
\begin{aligned}
\mathrm{G}_{\mathrm{IA}}(s) & =\mathrm{h}_{\mathrm{S}, \mathrm{IA}}\left(\mathrm{G}_{\mathrm{B}}(s)\right) \\
& \Rightarrow \mathrm{G}_{\mathrm{B}}(s)=\mathrm{h}_{\mathrm{S}, \mathrm{IA}}^{-1}\left(\mathrm{G}_{\mathrm{IA}}(s)\right) \\
\mathrm{G}_{\mathrm{IP}}(s) & =\mathrm{h}_{\mathrm{S}, \mathrm{IP}}\left(\mathrm{G}_{\mathrm{P}}(s)\right)=\mathrm{h}_{\mathrm{S}, \mathrm{IP}}\left(\mathrm{H}_{\mathrm{P}}(s) \mathrm{G}_{\mathrm{B}}(s)\right) \\
& \Rightarrow \mathrm{G}_{\mathrm{B}}(s)=\frac{\mathrm{h}_{\mathrm{S}, \mathrm{IP}}-1\left(\mathrm{G}_{\mathrm{IP}}(s)\right)}{\mathrm{H}_{\mathrm{P}}(s)}
\end{aligned}
$$



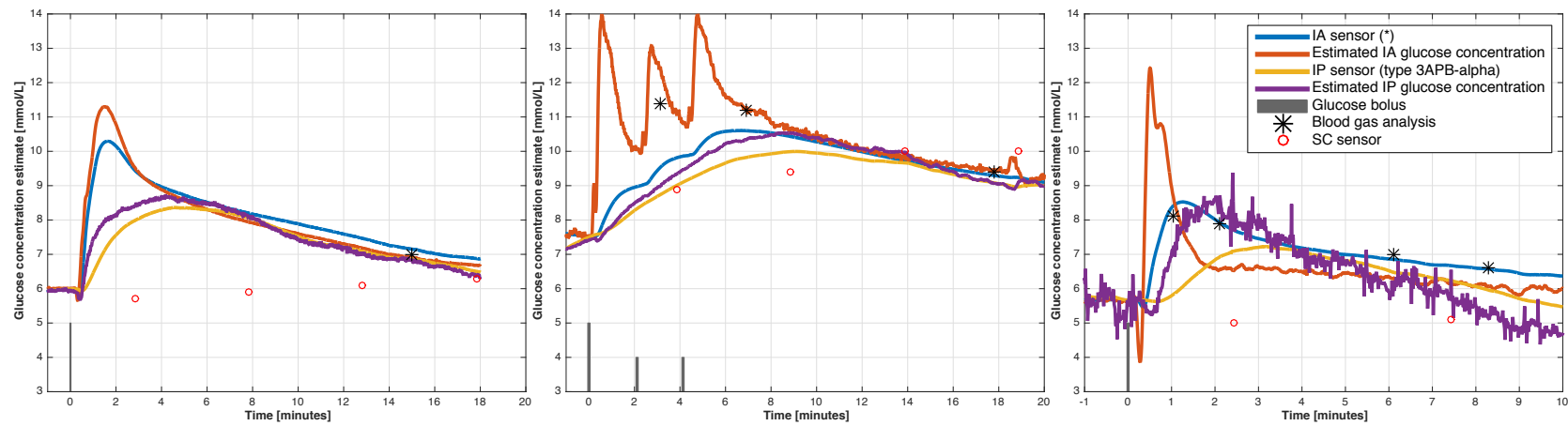

(a) Segment 1A, with a glucose bolus of 2(b) Segment 2A, with a glucose bolus series(c) Segment 3E, with a glucose bolus of 2 g. of 2,1 and $1 \mathrm{~g}$, respectively.

g.

Figure 4: Examples of intraarterial (IA) and intraperitoneal (IP) sensor signals and corresponding IA and IP glucose concentration estimates for one segment of each animal experiment. Beware that the sensor signals are uncalibrated and thus have no unit. $\left(^{*}\right)$ The IA sensor type was 3ABP-beta in experiment 2 and 3ABP-alpha in experiments 1 and 3, as described in Section 2.2. Copyright: CC BY-NC-SA 4.0 .

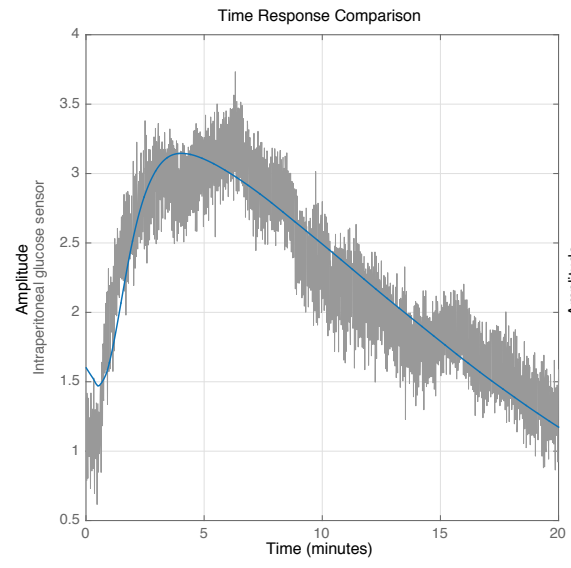

(a) Segment 1D.

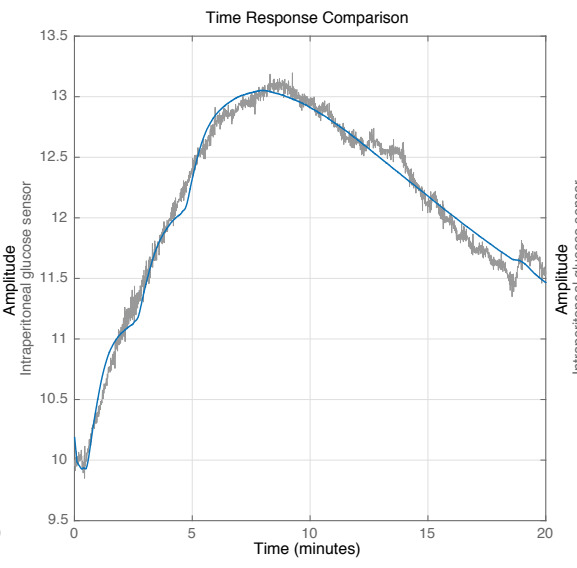

(b) Segment 2A.

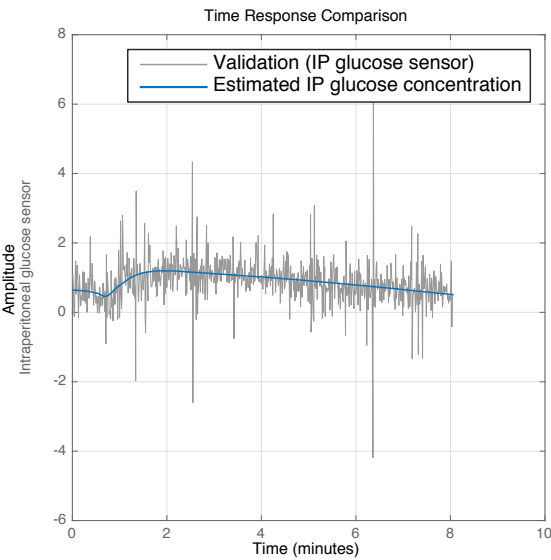

(c) Segment 3A.

Figure 5: Examples of identification of arterial to peritoneal glucose dynamics, i.e. the transfer function $\mathrm{H}_{\mathrm{P}}(s)$, for one segment of each animal experiment. The grey signal represents the IP sensor signal, while the blue signal shows the IA sensor signal transferred through $\mathrm{H}_{\mathrm{P}}(s)$. Beware of the scaling of the vertical axes, and that they have no unit, since the signals are uncalibrated. The seemingly variable signal-to-noise ratio is mostly due to the scaling. Copyright: CC BY-NC-SA 4.0.

Combining Eqs. 1 and 2 gives Eq. 3:

$$
\frac{\mathrm{h}_{\mathrm{S}, \mathrm{IP}}^{-1}\left(\mathrm{G}_{\mathrm{IP}}(s)\right)}{\mathrm{H}_{\mathrm{P}}(s)}=\mathrm{h}_{\mathrm{S}, \mathrm{IA}}^{-1}\left(\mathrm{G}_{\mathrm{IA}}(s)\right)
$$

For each segment of the experiments, the parameters of $\mathrm{H}_{\mathrm{P}}(s)$ were estimated by minimization of normalized root mean square error (NRMSE), using the System Identification Toolbox of Matlab R2015a (Mathworks, Inc., MA, USA). The resulting values of $\mathrm{T}$ and $\tau$ for each experiment segment are reported along with the NRMSE fitness value [\%] in Table 1. The "fitness value" is 1 - NRMSE, i.e. $100 \%$ corresponds to a perfect fit.

In addition to Matlab's system identification methods, manual adjustments of $\tau$ and the initial value for $\mathrm{T}$ were used for all segments in order to ensure that the identified transfer function yielded acceptable glucose concentration estimates (i.e. ensuring a result sufficiently close to the global optimum, rather than a local optimum). 


\subsubsection{Sensor dynamics}

In order to identify $\mathrm{H}_{\mathrm{P}}(\mathrm{s})$, the sensor dynamics had to be compensated. In practice, the sensor dynamics were inverted at both IP and IA sites before the system was identified, resulting in Eq. 4 based on Eq. 3:

$$
\mathrm{G}_{\mathrm{P}}(s)=\mathrm{H}_{\mathrm{P}}(s) \mathrm{G}_{\mathrm{B}}(s)
$$

where $\mathrm{G}_{\mathrm{P}}(s)=\mathrm{h}_{\mathrm{S}, \mathrm{IP}}^{-1}\left(\mathrm{G}_{\mathrm{IP}}(\mathrm{s})\right)$ and $\mathrm{G}_{\mathrm{B}}(s)=$ $\mathrm{h}_{\mathrm{S}, \mathrm{IA}}^{-1}\left(\mathrm{G}_{\mathrm{IA}}(s)\right)$, which requires that the sensor dynamics are invertible. The sensor dynamics are described by the nonlinear differential equation:

$$
\begin{aligned}
\frac{d \Delta \mathrm{L}(\mathrm{t})}{d t}= & \frac{1}{\mathrm{~T}_{0}}\left(\mathrm{~K}_{\mathrm{eq}} \Delta \mathrm{L}_{\max } \mathrm{G}_{\mathrm{loc}}(t)\right. \\
& \left.-\left(1+\mathrm{K}_{\mathrm{eq}} \Delta \mathrm{L}_{\max }\right) \mathrm{G}_{\mathrm{loc}}(t)\right)
\end{aligned}
$$

where $\mathrm{G}_{\text {loc }}(t)$ is the glucose concentration at the sensor location (i.e. $\mathrm{G}_{\mathrm{B}}(t)$ for the IA sensor and $\mathrm{G}_{\mathrm{P}}(t)$ for the IP sensor), $\Delta \mathrm{L}(t)$ is the sensor signal (i.e. $\mathrm{G}_{\mathrm{IA}}(t)$ for the IA sensor and $\mathrm{G}_{\mathrm{IP}}(t)$ for the IP sensor) reflecting a length change in the gel caused by the glucose concentration in the surrounding fluid, $\mathrm{K}_{\mathrm{eq}}$ is an equilibrium constant, $\Delta L_{\max }$ is the maximum value of $\Delta \mathrm{L}(t)$, and $\mathrm{T}_{0}$ is the characteristic time constant. The values of $\mathrm{K}_{\mathrm{eq}}$ and $\Delta L_{\max }$ are extracted from the static parts of the 3 -point calibration, i.e. when the signal has stabilized after placing the sensor in each of the 3 solutions. $\mathrm{T}_{0}$ is extracted from a transient sensor response, also during the 3 -point calibration. The identified parameter sets for all sensors are reported in Table 2 .

Eq. 5 can be solved with respect to $\mathrm{G}_{\text {loc }}(t)$ to yield:

$$
\mathrm{G}_{\mathrm{loc}}(\mathrm{t})=\frac{1}{\mathrm{~K}_{\mathrm{eq}} \Delta L_{\max }} \frac{\Delta \mathrm{L}(\mathrm{t})+\mathrm{T}_{0} \frac{d \Delta \mathrm{L}(\mathrm{t})}{d t}}{1-\frac{\Delta \mathrm{L}(\mathrm{t})}{\Delta L_{\max }}}
$$

Since the sensor dynamics described by Eq. 5 is minimum-phase, it can be inverted, but the inversion amplifies the high-frequent noise due to the differentiation of the sensor signal. The original sensor signals and estimated IA and IP glucose concentration for one segment of each experiment are presented in Figure 4. The inverted signals are smoothed by zero-phase averaging over 5 samples, but only for the illustrations (i.e. not for the input to system identification).

\subsubsection{Calculation of $t_{\max / 2}$}

For all segments of the trial containing a single glucose bolus, the time to reach half of the maximum IP sensor signal (denoted $t_{\max / 2}$ ) was calculated. Since the baseline was unknown, a model of the dynamics from the glucose bolus to the IP glucose sensor signal was identified for each segment in order to find a better estimate

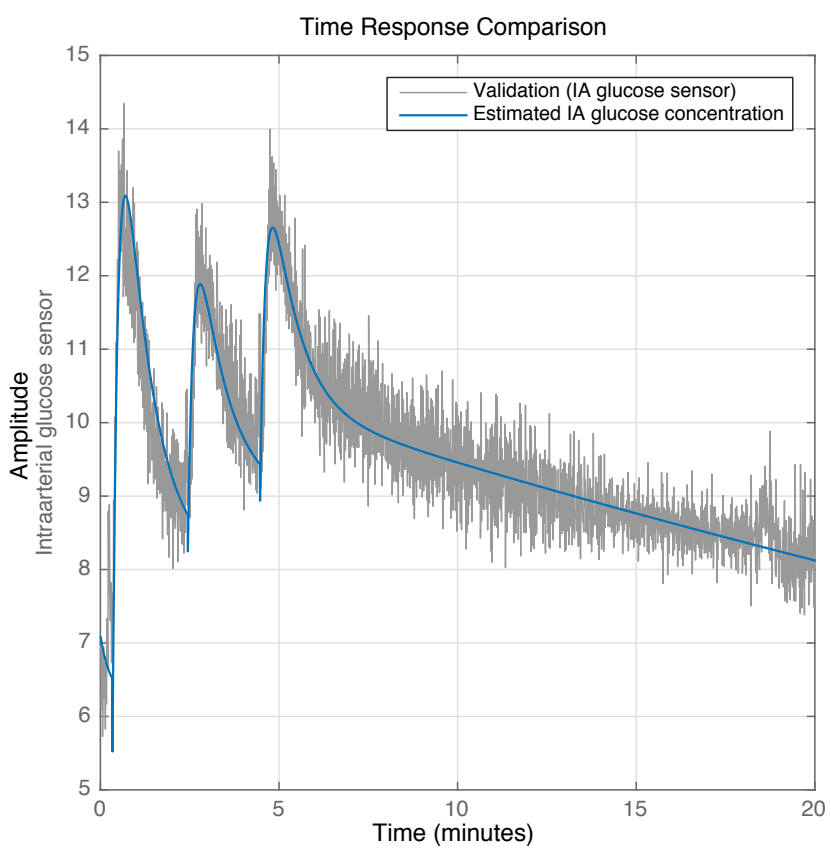

Figure 6: Example of identification of the dynamics $\mathrm{H}_{\mathrm{C}}(s) \mathrm{h}_{\mathrm{S}, \mathrm{IA}}(\cdot)$ from the glucose bolus to the arterial glucose concentration for segment 2A. The grey signal represents the IA sensor signal, while the blue signal shows a glucose bolus transferred through $\mathrm{H}_{\mathrm{C}}(s) \mathrm{h}_{\mathrm{S}, \mathrm{IA}}(\cdot)$. Beware that the vertical axis has no unit, since the signals are uncalibrated. Copyright: CC BY-NC-SA 4.0.

of $t_{\max / 2}$. This corresponds to $\mathrm{H}_{\mathrm{C}}(s) \mathrm{H}_{\mathrm{P}}(s) \mathrm{h}_{\mathrm{S}, \mathrm{IP}}(\cdot)$ in Figure 3. For experiment 1 and 3 the sensor types at the IA and IP locations were identical, so one can use $\mathrm{H}_{\mathrm{C}}(s) \mathrm{h}_{\mathrm{S}, \mathrm{IP}}(\cdot)=\mathrm{H}_{\mathrm{C}}(s) \mathrm{h}_{\mathrm{S}, \mathrm{IA}}(\cdot)$, representing the dynamics from the glucose bolus to the IA glucose sensor signal. These dynamics were modelled by a time delay and a transfer function with 2 zeros and 3 poles, which gave satisfactory fit. The resulting fit values, time delays and $t_{\max / 2}$ are reported in Table 1 . For experiment 2 , these values are not reported, since only multi-bolus series were included in that experiment.

\subsubsection{Protocol adjustments}

As mentioned above, the protocol was adjusted during the study. In summary the changes are: 1) More frequent BG samples/analysis after glucose boluses in experiment 2 and 3. 2) More IP sensors were used in experiment 2 than in experiment 1 , in order to test more IP sensor locations and to have a working sensor if some of them failed. 3) Fewer IP sensors were used in experiment 3 than experiment 2 due to lack of resources to handle them during the experiment. 
Fougner et.al., "Intraperitoneal Glucose Sensing is Sometimes Surprisingly Rapid"

Table 1: Results summarized. The bottom part shows the median value, mean value and standard deviation for the identified parameters.

\begin{tabular}{|c|c|c|c|c|c|c|c|c|}
\hline \multirow[t]{2}{*}{ Segment } & \multicolumn{3}{|c|}{ IA to IP dynamics } & \multicolumn{2}{|c|}{ Bolus to IA dynamics } & \multicolumn{3}{|c|}{ Bolus to IP dynamics } \\
\hline & Delay $[s]$ & Time constant [min] & Fit $^{1}$ & Delay $[\mathrm{s}$ & Fit $^{1}$ & Delay $[\mathrm{s}]$ & Fit $^{1}$ & $t_{\max / 2}[\mathrm{~s}]$ \\
\hline $1 \mathrm{~A}$ & 0 & 2.2 & $66.1 \%$ & 19.0 & $92.4 \%$ & 19.0 & $71.5 \%$ & 84.6 \\
\hline $1 B$ & 0 & 9.0 & $72.5 \%$ & 23.0 & $93.6 \%$ & 23.0 & $73.7 \%$ & 127.8 \\
\hline $1 \mathrm{C}$ & 0 & 5.5 & $54.1 \%$ & 21.0 & $93.1 \%$ & 21.0 & $55.1 \%$ & 112.8 \\
\hline 1D & 0 & 4.8 & $70.9 \%$ & 23.0 & $94.0 \%$ & 23.0 & $72.3 \%$ & 100.2 \\
\hline $2 \mathrm{~A}$ & 6.0 & 4.9 & $87.6 \%$ & 18.0 & $59.4 \%$ & \multicolumn{3}{|c|}{ Bolus series $^{2}$} \\
\hline $2 \mathrm{~B}$ & 0.0 & 6.6 & $10.9 \%$ & 22.0 & $76.8 \%$ & \multicolumn{3}{|c|}{ Bolus series $^{2}$} \\
\hline $3 \mathrm{~A}$ & 18.0 & 6.4 & $6.1 \%$ & 9.0 & $77.5 \%$ & 27.0 & $6.2 \%$ & 65.4 \\
\hline $3 \mathrm{~B}$ & 22.0 & 3.6 & $21.5 \%$ & 22.0 & $70.5 \%$ & 44.0 & $21.6 \%$ & 99.0 \\
\hline $3 \mathrm{C}$ & 15.0 & 10.2 & $19.8 \%$ & 18.0 & $78.5 \%$ & 33.0 & $19.8 \%$ & 171.0 \\
\hline $3 \mathrm{D}$ & 19.0 & 1.3 & $10.2 \%$ & 20.0 & $70.4 \%$ & 39.0 & $9.9 \%$ & 83.4 \\
\hline $3 \mathrm{E}$ & 12.0 & 3.4 & $47.1 \%$ & 18.0 & $74.5 \%$ & 30.0 & $47.3 \%$ & 69.6 \\
\hline $3 \mathrm{~F}$ & 8.5 & 2.2 & $41.5 \%$ & 20.0 & $69.5 \%$ & \multicolumn{3}{|c|}{ Bolus series $^{2}$} \\
\hline $3 \mathrm{G}$ & 26.0 & 0.5 & $22.8 \%$ & 11.8 & $52.5 \%$ & \multicolumn{3}{|c|}{ Bolus series $^{2}$} \\
\hline Median & 8.5 & 4.8 & $47.1 \%$ & 20.0 & $76.8 \%$ & 27.0 & $47.3 \%$ & 99.0 \\
\hline Mean & 9.7 & 4.7 & $45.5 \%$ & 18.8 & $77.1 \%$ & 28.8 & $41.9 \%$ & 101.5 \\
\hline St.dev. & 9.5 & 2.9 & $27.2 \%$ & 4.2 & $13.3 \%$ & 8.5 & $27.9 \%$ & 32.8 \\
\hline
\end{tabular}

${ }^{1}$ Fit is defined as $1-N R S M E$ (normalized root mean square error), i.e. $100 \%$ is perfect fit, as described in Section 2.

${ }^{2}$ Segments 2A, 2B, 3F and 3G contained bolus series and have thus no calculation of "Bolus to IP dynamics" or $t_{\max / 2}$.

\section{Results}

Figure 4 shows examples of sensor dynamics inversion and the resulting IA and IP glucose level estimates for segments $1 \mathrm{~A}, 2 \mathrm{~A}$ and $3 \mathrm{E}$. The relatively large difference between IA sensor signal and IA glucose concentration estimate in experiment 2 demonstrates the slow sensor dynamics of the 3ABP-beta sensors and confirms that the glucose estimates become similar to those experienced with the normal 3ABP-alpha sensors in experiments 1 and 3 . It can also be seen that the IP glucose concentration estimates of experiment 3 were noisier than in the other experiments.

Figure 5 shows examples of system identification for the IA to IP dynamics, $\mathrm{H}_{\mathrm{P}}(s)$, for segments $1 \mathrm{D}, 2 \mathrm{~A}$ and $3 \mathrm{~A}$. These are considered to be representative of the overall experiment. Segment $3 \mathrm{~A}$ shows that even with a relatively low fit value of $6.1 \%$ (see Table 1 ), the resulting estimate is reasonable.

Figure 6 and Figure 7 show examples of system identification for the dynamics from the glucose bolus to the IA and IP sensor signals, respectively, for segments $2 \mathrm{~A}$ and $3 \mathrm{C}$. These segments are selected as a good representation of the overall result.

Table 1 summarizes the identified delays, time constants and goodness of fit (NRMSE fitness value) for each segment of the three animal experiments. It shows also the identified dynamics from the glucose bolus infusion to the IA and IP sensor signals, and the corresponding $t_{\max / 2}$, for the single glucose bolus segments. The bottom part shows median value, mean value and standard deviation for the identified parameters.

Table 2 shows sensor parameter sets for all the working IA and IP sensors.

\section{Discussion}

This proof of concept trial has demonstrated that glucose in peritoneal fluid may react almost two times faster to changes in the IA glucose levels than previously shown by Burnett et al. (2014). The mean time constant was here found to be 4.7 (SD 2.9) $\mathrm{min}$ compared to 5.6 (SD 2.9) min in Burnett's study, the mean time delay was found to be 9.7 (SD 9.5) s compared to 40.8 (SD 34.8) s in Burnett's study. Regarding the number of glucose excursions in these experiments, successful results were reported from 13 (out of 21) segments in 3 animals, while Burnett et al. (2014) reported that they successfully carried out 13 segments in 8 animals; without mentioning the number of rejected segments. As such, the two studies are comparable in 


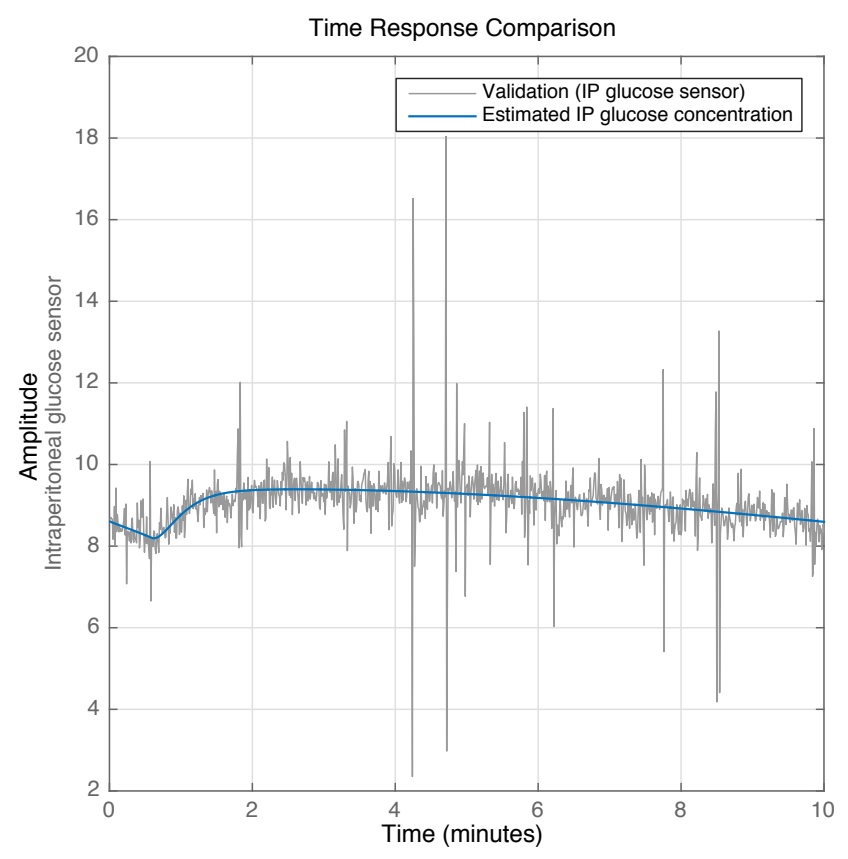

Figure 7: Example of identification of the dynamics $\mathrm{H}_{\mathrm{C}}(s) \mathrm{H}_{\mathrm{P}}(s) \mathrm{h}_{\mathrm{S}, \mathrm{IP}}(\cdot)$ from the glucose bolus to the peritoneal glucose concentration for segment 3C. The grey signal represents the IA sensor signal, while the blue signal shows a glucose bolus transferred through $\mathrm{H}_{\mathrm{C}}(s) \mathrm{H}_{\mathrm{P}}(s) \mathrm{h}_{\mathrm{S}, \mathrm{IP}}(\cdot)$. Beware that the vertical axis has no unit, since the signals are uncalibrated. Copyright: CC BY-NC-SA 4.0.

size. A possible cause of faster dynamics in our trials is that we did not include sensor dynamics in our calculations, since the sensor dynamics are separate from the physiology and should be excluded when characterizing the intrinsic properties of the human body.

The "IA to IP dynamics" system identification procedure performs well. Although the reported fit values are moderate, with a mean fit value of $45.5 \%$ (SD $27.2 \%$ ), it can be seen in Table 1 and the example plot for Segment 3A in Figure 5 that the low fit values originate mostly from the noisy IP glucose concentration estimates in experiment 3. In order to get satisfactory results, a manual optimization of delays and initial time constants was needed; otherwise the algorithm terminated at local optima with much lower fit values, as mentioned above. This can be interpreted as a weakness of the algorithms, but it is generally common in system identification to require some manual optimization when local minima are present.

When giving IV glucose boluses it was anticipated that the increase in circulatory glucose would reach the femoral artery (IA sensor) and the capillaries in the peritoneal lining at approximately the same time. However, that the measured IP glucose level should start to increase more or less concomitant with the glucose level in the peritoneal lining was a surprise. This indicates that IP sensors, at least the one in experiment 1, may have been measuring directly in contact with the capillaries in the peritoneal lining, i.e. with just a thin layer (consisting of little more than the capillary wall and the sensor membrane) between blood and the sensor element, leading to a negligible transport delay through IP fluid diffusion or convection.

In the present study, glucose boluses were used. A glucose bolus effectively is an impulse, which is better suited for identification purposes than slower glucose changes. This is because an impulse is richer in its frequency content and thus excites a wider range of dynamic modes in the system. In future experiments, a continuous IV glucose infusion given in a manner to imitate glucose excursions following a meal, may be used. This would document in a more lifelike manner whether or not IP glucose sensing is a realistic alternative in humans when designing an artificial pancreas.

A limitation of this study is that several sensors were damaged or gave non-interpretable signals. However, one sensor worked properly in each experiment and was analysed further. The sensors were placed in different intraperitoneal locations without detailed knowledge about the exact locations, but at least with an approximate location (the previous study by Burnett et al. (2014) does not contain any information about IP sensor placement). Anyway, it is currently not possible to know why some of the sensors had a rapid and consistent response, while other sensors had inconsistent or slow responses. The list of possible reasons is long; some sensors may have been stuck in adipose/fatty tissue, and some sensors may have been in contact with moving organs such as the intestine, which could result in noisy sensor signals. Some sensors may have been measuring in IP fluid, while other sensors may have measured directly on the peritoneal lining or the greater omentum with high density of capillaries. Further, the GlucoSet sensors are originally designed for intravascular use (Tierney et al., 2009). When placed in peritoneum they are more susceptible to mechanical strain/deformation during insertion or use, which may explain some of the discrepancies. It is therefore not a surprise that large variations were observed in the sensor responses. The focus on these possible confounders is an obvious aim in future experiments and essential to clarify if IP glucose sensing should become a clinical alternative in humans. These interferometric sensors were anyway used in this study because: a) They have fast response and documented features (Tierney et al., 2009), b) the sensors are developed by 
Fougner et.al., "Intraperitoneal Glucose Sensing is Sometimes Surprisingly Rapid"

Table 2: Sensor types and parameter sets.

\begin{tabular}{|l|c|l|c|c|c|}
\hline Sensor location & Experiment & Sensor type & $\Delta L_{\max }[\mathrm{nm}]$ & $1 / \mathrm{K}_{\mathrm{eq}}[\mathrm{mM}]$ & $\mathrm{T}_{0}[\mathrm{~s}]$ \\
\hline & 1 & 3APB-alpha & 11624 & 5.0 & 65 \\
IA, femoral artery & 2 & 3APB-beta & 14700 & 24.0 & 421 \\
& 3 & 3APB-alpha & 9965 & 3.2 & 100 \\
\hline IP, $15 \mathrm{~cm}$ into upper right quadrant & 1 & 3APB-alpha & 15851 & 14.8 & 83 \\
IP, 10 cm into lower right quadrant & 2 & 3APB-alpha & 11392 & 10.5 & 111 \\
IP, $15 \mathrm{~cm}$ into upper right quadrant & 3 & 3APB-alpha & 12559 & 17.1 & 104 \\
\hline
\end{tabular}

a spin-off company from the university the authors are affiliated with, c) they are designed for sensing in body fluids similar to peritoneal fluid, and d) there are no alternative sensors on the market that are designed for IP use.

In all segments, and especially visible in experiment 3 , the bolus infusions caused a small negative dip in both IA and IP sensor signals. This is probably a temperature effect; the glucose infusions were at room temperature $\left(20-22^{\circ} \mathrm{C}\right)$ and the sensors are sensitive to temperature. In future, this artifact may thus be avoided by using glucose boluses at body temperature. Assuming that it affected equally the IA and IP sensors, its effect on the identification of $\mathrm{H}_{\mathrm{P}}(\mathrm{s})$ in the presented study is negligible.

In experiment 2, and thereby in 2 out of the 13 reported segments, a slightly different sensor type was used at the IA location. The results do not differ much if only the 11 segments with identical sensor types are included. In other words, it does not affect the conclusions of the paper.

Calibration of the IP sensors to peritoneal fluid was not performed. Some IP fluid was sampled and analysed ex vivo before bolus infusions were initiated in experiment 1 , and the glucose value of this sample was consistent with the blood sample taken at the same time, but there was not enough IP fluid for more sampling without disturbing or possibly harming the sensors (e.g. by pushing and squeezing the animal's abdominal region in order to collect fluid) as well as the animal itself and possibly the dynamics to investigate in the experiment. Thus, in general there was a lack of a suitable reference for the IP sensors. They were only carried through a three-point calibration before insertion into peritoneum. The amplitudes and offsets of the IP sensor signals of this experiment were manually set. Thus, the absolute accuracy of the sensors cannot be analysed, and only the time response has been reported. However, knowledge about this time response is valuable as it demonstrates the intrinsic rapid glucose sensing in peritoneum independently of the sensor itself.

These experiments were performed in pigs. Since pigs and humans have different anatomy and physiol- ogy, one cannot yet conclude about how the IP sensor response will perform in humans. However, we have seen that in pigs, IP sensors may have a substantially faster response than SC sensors - faster than previously reported by Burnett et al. (2014). Even if dynamics are different in humans, one may expect that IP response will be faster than SC response even in humans. Clinical trials in humans are needed to confirm this.

Glucose dynamics may differ between different parts of the peritoneum. Peritoneal fluid is believed to be produced in the lower part of peritoneum and absorbed mainly in the upper part close to diaphragma (Patel and Planche, 2013), which could explain why our study (and the previous study (Burnett et al., 2014)) shows variable dynamics. Moreover, in the present study we observed negligible time delays both in the lower and upper right quadrant. This result may have two interpretations: 1) that the present understanding of the peritoneal fluid only being secreted in the lower peritoneal cavity is questionable, or 2) that a significant part of the intraperitoneal glucose is secreted directly through the peritoneal lining. It is also unclear whether the IP sensors were actually measuring concentrations representative for the peritoneal fluid or rather at direct diffusion from capillaries in visceral or parietal peritoneal lining. It is challenging to take samples for reference analysis from any of these sites, and thus there is currently a lack of a proper calibration method.

The sensors used in the IA and IP locations were designed for use in blood/fluids and cannot at present be inserted in the SC space without getting destroyed. Thus, we had to use a different sensor type for the $\mathrm{SC}$ location. Accordingly, due to the different sensors used, the SC sensor readings could not be used in system identification and calculation of $t_{\max / 2}$. In the present study the $\mathrm{SC}$ sensor readings can be considered as an illustration of the qualitative difference between SC and IP glucose dynamics.

The fast IP glucose dynamics reported in the present study is relevant when considering the pros and cons of different approaches for an artificial pancreas. One can claim that insulin absorption dynamics is more important than sensing dynamics for the closed-loop system. 
In other words, it would be more beneficial to focus on faster insulin analogues and new insulin infusion locations, than to focus on the sensor location. However, we hold that both the insulin absorption dynamics and the sensing dynamics must be improved in order to construct a robust artificial pancreas able to normalize or near normalize glucose levels in patients with diabetes. The major value of the present pilot study is that it indicates that IP glucose sensing may be nearly as fast as the sensing of glucose levels by beta cells in individuals without diabetes.

\section{Conclusions}

In spite of variations in the execution of the experiments were performed (due to the minor protocol adjustments described before), the results of these experiments are consistent across various sensors, procedures and animals. It is a strong indication of faster glucose dynamics between blood and peritoneum than shown in previous studies and is encouraging for the use of IP glucose sensing in an artificial pancreas, where one of the main open challenges is to achieve sufficiently fast dynamics in the glucose control loop.

\section{Acknowledgments}

Oddveig Lyng at the Unit of Comparative Medicine, NTNU, and Tine A. Hunt and Sondre Volden of GlucoSet AS, are acknowledged for their invaluable contributions to the animal experiments. Olav Spigset at Dept. of Clinical Pharmacology, St Olavs Hospital, is acknowledged for contribution to the study design.

\section{Disclosures}

An abstract and a poster with preliminary results from this paper were presented at the ATTD conference in Paris, February 2015. Author N. A. Elvemo is general manager of GlucoSet AS, a company in the glucosemonitoring field. Author R. Ellingsen is board member of GlucoSet AS. Authors N. A. Elvemo, D. R. Hjelme and R. Ellingsen are shareholders of GlucoSet AS. Author S. M. Carlsen is advisory board member for Prediktor Medical AS, another company in the glucosemonitoring field. All authors except N. A. Elvemo are members of the Artificial Pancreas Trondheim (APT) research group at the Norwegian University of Science and Technology (NTNU), Trondheim, Norway.

\section{References}

Bailey, T. S., Chang, A., and Christiansen, M. Clinical accuracy of a continuous glucose monitoring system with an advanced algorithm. Journal of Diabetes Science and Technology, 2015. 9(2):209-214. doi:10.1177/1932296814559746.

Basu, A., Dube, S., Slama, M., Errazuriz, I., Amezcua, J. C., Kudva, Y. C., Peyser, T., Carter, R. E., Cobelli, C., and Basu, R. Time lag of glucose from intravascular to interstitial compartment in humans. Diabetes, 2013. 62(12):4083-4087. doi:10.2337/db131132.

Boyne, M. S., Silver, D. M., Kaplan, J., and Saudek, C. D. Timing of changes in interstitial and venous blood glucose measured with a continuous subcutaneous glucose sensor. Diabetes, 2003. 52(11):27902794. doi:10.2337/diabetes.52.11.2790.

Burnett, D., Huyett, L., and Zisser, H. Glucose sensing in the peritoneal space offers faster kinetics than sensing in the subcutaneous space. Diabetes, 2014. 63(7):2498-2505. doi:10.2337/db13-1649.

Cengiz, E. and Tamborlane, W. V. A tale of two compartments: Interstitial versus blood glucose monitoring. Diabetes Technology \& Therapeutics, 2009. 11(s1). doi:10.1089/dia.2009.0002.

Clark, L. C., Noyes, L. K., Spokane, R. B., Sudan, R., and Miller, M. L. Long-term implantation of voltammetric oxidase-peroxide glucose sensors in the rat peritoneum. Methods in Enzymology, 1988. doi:10.1016/0076-6879(88)37008-4.

Clark, L. C., Spokane, R. B., Sudan, R., and Stroup, T. L. Long-lived implanted silastic drum glucose sensors. Transactions - American Society for Artificial Internal Organs, 1987. 33.

Davey, R. J., Low, C., Jones, T. W., and Fournier, P. A. Contribution of an intrinsic lag of continuous glucose monitoring systems to differences in measured and actual glucose concentrations changing at variable rates in vitro. Journal of Diabetes Science and Technology, 2010. 4(6):1393-1399. doi:10.1177/193229681000400614.

Garcia, A., Rack-Gomer, A. L., Bhavaraju, N. C., Hampapuram, H., Kamath, A., Peyser, T., Facchinetti, A., Zecchin, C., Sparacino, G., and Cobelli, C. Dexcom g4ap: an advanced continuous glucose monitor for the artificial pancreas. Journal of Diabetes Science and Technology, 2013. 7(6):14361445. doi:10.1177/193229681300700604. 
Helton, K. L., Ratner, B. D., and Wisniewski, N. A. Biomechanics of the sensor-tissue interface - effects of motion, pressure, and design on sensor performance and foreign body response - part ii: Examples and application. Journal of Diabetes Science and Technology, 2011. 5(3):647-656. doi:10.1177/193229681100500318.

Mensh, B. D., Wisniewski, N. A., Neil, B. M., and Burnett, D. R. Susceptibility of interstitial continuous glucose monitor performance to sleeping position. Journal of Diabetes Science and Technology, 2013. 7(4):863-870. doi:10.1177/193229681300700408.

Patel, R. and Planche, K. Applied Peritoneal Anatomy. Clinical Radiology, 2013. 68(5):509-520.

Skjærvold, N. K., Lyng, O., Spigset, O., and Aadahl, P. Pharmacology of intravenous insulin administration: Implications for future closed-loop glycemic control by the intravenous/intravenous route. Diabetes Technology $\&$ Therapeutics, 2012. 14(1):23-29. doi:10.1089/dia.2011.0118.

Skjærvold, N. K., Östling, D., Hjelme, D. R., Spigset, O., Lyng, O., and Aadahl, P. Blood glucose control using a novel continuous blood glucose monitor and repetitive intravenous insulin boluses: Exploiting natural insulin pulsatility as a principle for a future artificial pancreas. International Journal of Endocrinology, 2013. 245152:1-8. doi:10.1155/2013/245152.

Stout, P. J., Racchini, J. R., and Hilgers, M. E. A novel approach to mitigating the physiological lag between blood and interstitial fluid glucose measurements. Diabetes Technology \& Therapeutics, 2004. 6(5):635-644. doi:10.1089/dia.2004.6.635.

Tierney, S., Falch, B. M. H., Hjelme, D. R., and Stokke, B. T. Determination of glucose levels using a functionalized hydrogel-optical fiber biosensor: Toward continuous monitoring of blood glucose in vivo. Analytical Chemistry, 2009. 81(9):3630-3636. doi:10.1021/ac900019k.

Velho, G., Froguel, P., and Reach, G. Determination of peritoneal glucose kinetics in rats, implications for the peritoneal implantation of closed-loop insulin delivery systems. Diabetologia, 1989. 32. 